\title{
Infrared scintillation yield in gaseous and liquid argon
}

\author{
A. Buzulutskov (a), A. Bondar, A. Grebenuk \\ Budker Institute of Nuclear Physics, Novosibirsk, 630090, Russia
}

\begin{abstract}
PACS 29.40.Mc - Scintillation detectors
PACS 78.60.Fi - Electroluminescence

PACS $61.25 . \mathrm{Bi}$ - Liquid noble gases
\end{abstract}

\begin{abstract}
The study of primary and secondary scintillations in noble gases and liquids is of paramount importance to rare-event experiments using noble gas media. In the present work, the scintillation yield in gaseous and liquid Ar has for the first time been measured in the near infrared (NIR) and visible region, both for primary and secondary (proportional) scintillations, using Geiger-mode avalanche photodiodes (G-APDs) and pulsed X-ray irradiation. The primary scintillation yield of the fast component was measured to be $1.7 \times 10^{4}$ photon/ $\mathrm{MeV}$ in gaseous $\mathrm{Ar}$ in the NIR, in the range of $690-1000 \mathrm{~nm}$, and $5.1 \times 10^{2}$ photon/MeV in liquid $\mathrm{Ar}$, in the range of 400-1000 nm. Proportional NIR scintillations (electroluminescence) in gaseous Ar have been also observed; their amplification parameter at $163 \mathrm{~K}$ was measured to be 13 photons per drifting electron per kV. Possible applications of NIR scintillations in high energy physics experiments are discussed.
\end{abstract}

Introduction. - The study of primary and secondary sintillations in noble gases and liquids is of paramount importance to rare-event experiments using noble gas media, in particular to those of dark matter search [1] 3 , $\mu$-e- $\gamma$ decay search [4], neutrinoless double beta decay search [5] and coherent neutrino-nucleus scattering [6, [7. For example, the difference in light yields of primary scintillations produced by nuclear recoils and $\gamma$-quanta in liquid Ar and Xe, provides an effective means for selecting WIMP signals against the background in dark matter search experiments using two-phase noble gas detectors [8, i.e. in ZEPLIN

-. 1], XENON [2] and WARP [3] experiments. In addition, $\geq$ to record the ionization signal along with that of the primary scintillation, secondary (proportional) scintillations áre used in such experiments, i.e. those of electroluminescence generated by electrons drifting under moderate electric field in the gas phase 9, 10. The other examples of noble gas scintillation applications are the noble liquid scintillation calorimetry, in particular that of the MEG experiment 4] based on recording primary scintillations in liquid Xe, and the high-pressure noble-gas TPC of the NEXT experiment [5] based on recording proportional scintillations in compressed Xe.

So far, noble gas scintillations in high energy physics experiments have been recorded essentially in the vacuum

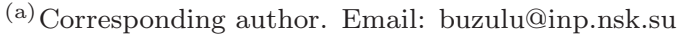

ultraviolet (VUV). This necessitated the use of sophisticated VUV-sensitive photodetectors, including those with Wavelength Shifter (WLS) coatings. Indeed, in the VUV the primary scintillation yield is rather high: of about (40$60) \times 10^{3}$ photon/MeV in liquid $\mathrm{Ar}$ and $\mathrm{Xe}$ [8 and $14 \times 10^{3}$ photon/MeV in gaseous Xe 11. The VUV emission is caused by reactions between excited and ionized atoms producing excimers which decay radiating the VUV continua [12. In addition in condensed noble gases, a mechanism of VUV luminescence due to excitation of excitons might play a role [13. At high pressures the VUV emission was generally believed to dominate over all other types of radiative decays such as atomic emission in the visible and infrared regions [8].

On the other hand, as early as 20 years ago it was suggested that this statement might not be valid due to the discovery of intense atomic emission scintillations in practically all noble gases in the near infrared (NIR) [14]: in particular in Ar in the wavelength range of $690-850 \mathrm{~nm}$ [14] and in Xe at 800-1600 nm [15. Since then, the NIR emission spectra of scintillations in gaseous and liquid $\mathrm{Ar}$ and Xe have been further studied in several works: both for primary [15,17] and secondary [18, 19] scintillations. In gaseous Ar this kind of scintillation was attributed to transitions between the atomic states of the $\operatorname{Ar}\left(3 p^{5} 4 p\right)$ and $\operatorname{Ar}\left(3 p^{5} 4 \mathrm{~s}\right)$ configurations [14, 18. However, little is known about the absolute NIR scintillation yield in noble 
gases: almost nothing about that in Ar and only the lower limit in gaseous $\mathrm{Xe}\left(\geq 21 \times 10^{3}\right.$ photon/MeV $)[19$.

In this paper we present the experimental data in support for the hypothesis of the intense NIR scintillations in $\mathrm{Ar}$ in view of its potential application in rare-event experiments: for the first time we have measured the scintillation yield in gaseous and liquid Ar in the NIR and visible region, using Geiger-mode Avalanche Photodiodes (G-APDs, [20]). The latter have rather high photon detection efficiency (PDE) in the wavelength range of 400-1000 $\mathrm{nm}$, of about $15 \%$ on average 21, providing direct and effective detection of NIR scintillations without WLS.

This work has actually been triggered by the recent results on NIR scintillations obtained in our laboratory 22]: a rather high avalanche (secondary) scintillation yield, of about 4 NIR photons per avalanche electron, was observed. This observation was done in the course of the development of a novel technique of signal recording in detectors of ultimate sensitivity for rare-event experiments, such as those of two-phase Ar avalanche detectors 23 based on thick Gas Electron Multipliers (THGEMs) 24 with optical readout using G-APDs 22, 25. We will discuss this technique in the following, as well as other possible applications of NIR scintillations in high energy physics experiments.

Experimental setup. - Fig. 1]shows the experimental setup; as concerns cryogenics and electronics it was similar to that described elsewhere 22. It consisted of a $9 \mathrm{l}$ cryogenic chamber filled with either gaseous or liquid Ar. Ar was taken from the bottle with a specified purity of $99.998 \%\left(\mathrm{~N}_{2}\right.$ content $\left.<10 \mathrm{ppm}\right)$; during cooling procedures it was additionally purified from oxygen and water by $\mathrm{Ox}$ isorb filter [26], providing electron life-time in the liquid $>20 \mu \mathrm{s}$ [27] which corresponds to the oxygen-equivalent impurity content below $20 \mathrm{ppb}$.

To cross-check the correctness of the scintillation yield measurement procedure, several measurement runs were conducted in a variety of ways: using two different photodetectors, at different solid angles, with and without NIR transmission optical filter. In particular, the scintillation and ionization signals were recorded in two different gaps, top and bottom, with an active area of $30 \times 30 \mathrm{~mm}^{2}$ and thickness of 2 and $10 \mathrm{~mm}$ respectively (see Fig.1). The gaps were formed by thin-plate electrodes made of copper-clad G10 and a wire grid as shown in Fig. 1.

The signals in the gaps were induced by X-rays from a pulsed X-ray tube with a Mo anode operated at a voltage of $40 \mathrm{kV}$. The gaps were irradiated from outside the cryogenic chamber, practically uniformly across the active area, through a lead collimator and aluminium windows at the chamber bottom, defining a cylindrical X-ray conversion region of a $20 \mathrm{~mm}$ diameter in each gap. Accordingly, the energies of incident X-rays were within 15-40 $\mathrm{keV}$ in gaseous $\mathrm{Ar}$ and $30-40 \mathrm{keV}$ in liquid Ar. The X-ray pulse had a sufficient power to provide measurable ionization charges in the gaps, typically having values of several

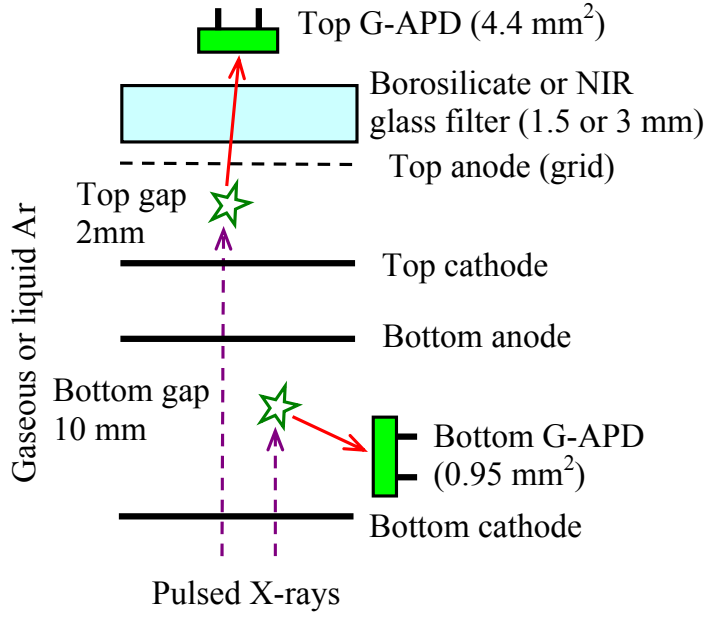

Fig. 1: Schematic view of the experimental setup to study scintillations in the NIR and visible region in gaseous and liquid Ar (not to scale).

thousands and tens of thousands electrons in gaseous and liquid Ar respectively. In addition it was sufficiently fast, having a width of $0.5 \mu \mathrm{s}$, to provide a reasonable scintillation time resolution.

The gaps were viewed by respectively top and bottom G-APDs as shown in Fig. 1 (manufactured by CPTA company [21]), namely by "CPTA 149-35" and "CPTA 14322 " having 4.41 and $0.95 \mathrm{~mm}^{2}$ sensitive area, respectively. The G-APDs were sensitive in the wavelength range of 400-1000 nm: at the gaseous Ar emission spectrum maximum (800 nm 14, 18]) they had PDE 15\% 21. In front of the top G-APD either a borosilicate glass filter, transparent to visible and NIR light, or NIR glass filter, transparent to NIR above $700 \mathrm{~nm}$, was placed. The bottom G-APD had no optical filter. It is essential that the measurements were carried out at cryogenic temperatures, in the range of 87-163 K, where G-APDs have a superior performance as compared to room temperature, in terms of the noise rate, amplitude and single-photoelectron characteristics 28.

The scintillation signal was read out from either the top or bottom G-APD using a fast amplifier (CPTA, 21]) with $300 \mathrm{MHz}$ bandwidth. The charge (ionization) signal was read out from the anode electrode of either the top or bottom gap using a charge-sensitive amplifier. Both amplifiers were placed outside the cryogenic chamber. The signals were digitized and memorized for further off-line analysis with a TDS5032B digital oscilloscope.

Measurement procedure. - It should be remarked that any measurement of the absolute scintillation yield is generally a difficult task, since one has to correctly determine the number of photons emitted in the scintillation burst. In the present work we applied a new approach to make such measurements, namely using the G-APD in single photoelectron counting mode with time resolution. As compared to other photodetectors, in addition to its higher 
sensitivity in the NIR, the G-APD provides a faster singlephotoelectron pulse of a well-defined shape, with a width of $20 \mathrm{~ns}$ [22,28]. These resulted in a remarkable capability of the G-APD to count the number of photoelectrons contained in a given (digitized) scintillation signal: by counting the number of single-photoelectron pulses in a time scale corresponding to the scintillation signal duration. To realize this method, the X-ray pulse intensity was reduced down to a level providing a practically non-overlapping of single-photoelectron pulses in the scintillation signal. This was the key element of the measurement procedure; the other details of the procedure were as follows.

The scintillation yield is defined as the ratio of the number of photons emitted over full solid angle $\left(N_{p h}\right)$ to the number of ionization electrons created in the gap $\left(N_{e}\right)$. In practice Y was obtained from the G-APD's photoelectron yield over full solid angle $\left(Y_{p e}\right)$ and the PDE averaged over the spectrum of the Ar emission passed through the optical filter $(<P D E\rangle)$ :

$Y=N_{p h} / N_{e}=Y_{p e} /\langle P D E\rangle$.

$<P D E\rangle$ was calculated using a G-APD's PDE spectrum provided by the manufacturer [21] and Ar emission spectrum taken from Refs. 14] and [17] for gaseous and liquid Ar respectively: $\langle P D E\rangle$ was equal to $17.6 \%$ and $16.7 \%$ respectively when using the borosilicate glass filter and to $12.6 \%$ when using the NIR filter.

$Y_{p e}$ was obtained from the charge signal amplitude $\left(N_{e}\right)$, scintillation signal amplitude $\left(N_{p e}\right)$ and G-APD average acceptance with respect to the $\mathrm{X}$-ray conversion region $\left(A_{G-A P D}\right)$, equal to the average reduced solid angle $(<$ $\left.\Delta \Omega_{G-A P D} / 4 \pi>\right)$ : $Y_{p e}=N_{p e} / N_{e} / A_{G-A P D}$.

$A_{G-A P D}$ was accurately calculated using Monte-Carlo simulation. In these calculations one took into account the light reflection from the electrodes for both gaps; in addition for the top gap, the light refraction, Frenel reflections and absorption in the optical filter, if any, as well as the anode grid transparency were taken into account. The calculated $A_{G-A P D}$ values amounted to 0.0029 for the top G-APD and 0.00018 for the bottom G-APD. Notice a considerable difference, of a more than an order of magnitude, in acceptances for the top and bottom G-APDs.

$N_{e}$ is just the ionization signal amplitude expressed in electrons; it was measured at the anode electrode under electric field applied across the gap. Similarly, $N_{p e}$ is the scintillation signal amplitude expressed in photoelectrons; it was measured using the method described above.

Regarding the measurement errors, the major sources of uncertainties were classified as follows: the $N_{e}$ and $N_{p e}$ measurements gave $10-30 \%$ and $10-20 \%$ respectively (depending on a particular measurement run), the $\langle P D E\rangle$ calculation gave $5 \%$. In total these resulted in about 15 $40 \%$ uncertainty in the scintillation yield.

Further details of the experimental setup and procedure will be presented in our more elaborated paper [29].

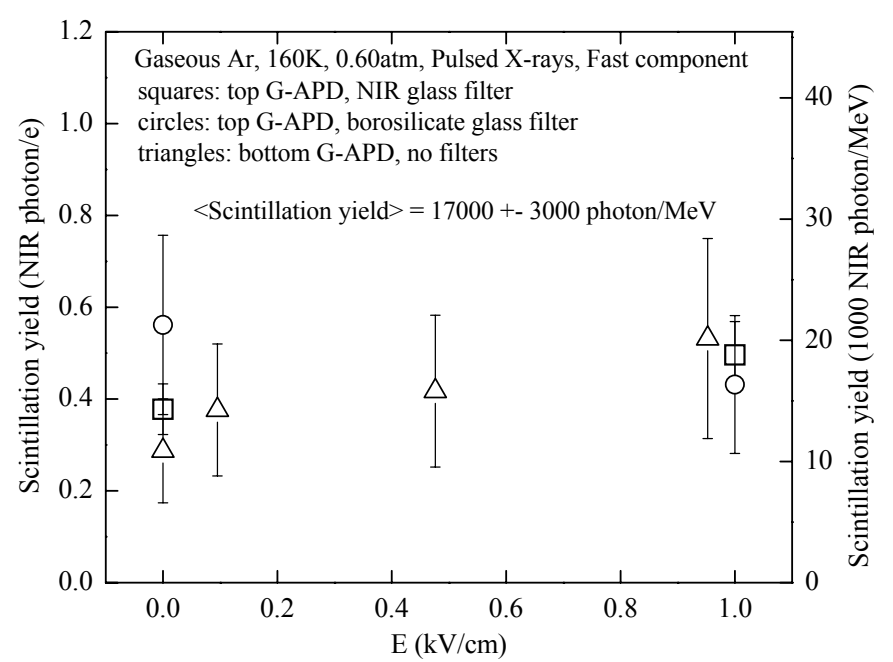

Fig. 2: Primary NIR scintillation yield for the fast component in gaseous $\mathrm{Ar}$ at $160 \mathrm{~K}$ and $0.60 \mathrm{~atm}$, measured in the electric field range of $0-1 \mathrm{kV} / \mathrm{cm}$ under different conditions: with top G-APD and NIR glass filter, with top G-APD and borosilicate glass filter, with bottom G-APD and no optical filter. The scintillation yield is expressed in the number of photons per primary ionization electron (left scale) and photons per $\mathrm{MeV}$ of deposited energy (right scale)

Results and discussion. - In this paper we present the results obtained at cryogenic temperatures: in gaseous Ar - at a temperature of about $160 \mathrm{~K}$ and pressure of 0.60 atm (with gas density corresponding to that of 1 atm at room temperature) and at $87 \mathrm{~K}$ and $1.0 \mathrm{~atm}$ (i.e. in saturated vapor); in liquid $\mathrm{Ar}$ - at $87 \mathrm{~K}$ and $1.0 \mathrm{~atm}$. The scintillations in the G-APD sensitivity region were observed under these conditions and their yields were measured in both gaseous and liquid Ar.

The scintillation signal had a fast component with a time structure reflecting that of the X-ray pulse, thus having a time constant below $0.5 \mu \mathrm{s}$. This time scale was expected from the atomic emission scintillation mechanism. On the other hand in addition to the fast component, a slow scintillation component was observed in both gaseous and liquid Ar, with a time constants of about $20 \mu \mathrm{s}$. Its integral contribution to the light yield, in a $50 \mu$ s time interval, was comparable to that of the fast component. The origin of the slow component has not been yet understood. Most probably it is caused by slow scintillations of Ar or those of impurities of yet unclear nature; other hypotheses such as delayed X-ray fluorescence, optical filter fluorescence and G-APD after-pulses were tested and practically excluded in supplementary measurements. Accordingly in the following, only the fast component contribution to the scintillation yield will be taken into consideration; the slow component contribution was accurately measured and subtracted.

In gaseous Ar, at low and moderate electric fields applied across the gap, varying from 0.1 to $4 \mathrm{kV} / \mathrm{cm}$, the gaps were operated in an ionization mode. i.e. without 
electron multiplication. At these fields the collected charge was practically independent of the field, enabling to unambiguously determine the primary ionization charge $\left(N_{e}\right)$. The primary scintillation yield was measured at low electric fields, i.e. in the absence of secondary scintillations, in the range of $0-1 \mathrm{kV} / \mathrm{cm}$ : it is shown in Fig. 2. Three groups of data are presented obtained under substantially different conditions: with the top G-APD and NIR glass filter, with the top G-APD and borosilicate glass filter, with the bottom G-APD and no optical filter.

One can see that there is a good agreement between all the data within their accuracies. Hence one may conclude the following. Firstly, the scintillations took place essentially in the NIR, since the scintillation yields obtained with and without the NIR filter were close to each other. Secondly, the NIR emission spectrum in our measurements is similar to that of Ref. 14, since just this spectrum provided the data compatibility. Thirdly, the acceptances were calculated correctly, since the data obtained in the top and bottom gaps are consistent, despite the considerable difference in measurement geometries and G-APD solid angles.

The primary NIR scintillation yield in gaseous Ar, averaged over all the measurements presented in Fig. 2, is $Y_{G A r}=0.44 \pm 0.09$ photon/e. It is convenient to express the yield in the number of photons per $\mathrm{MeV}$ of deposited energy considering a W-value (energy needed to produce one ion pair) in gaseous $\mathrm{Ar}$ of $26.4 \mathrm{eV}$ [8]: $Y_{G A r}=17000 \pm 3000$ photon/MeV. It should be remarked that this primary scintillation yield was found to be independent of the temperature in the range of 87-163 K. It is also remarkable that this yield value is close to that of the VUV for gaseous Xe [11 and to that of the low limit established for NIR scintillations in Xe [19. Thus, one may conclude that the primary scintillation yield is rather similar for all noble gases, both in the VUV and NIR regions.

In liquid Ar, the charge collected in the gap $\left(N_{c}\right)$ depends on the electric field $(E)$ due to the recombination effect. In our measurements this dependence was described in the frame of the recombination model of Ref. [9] enabling to determine the primary ionization charge $\left(N_{e}\right)$ : $N_{c}=N_{e} /(1+k / E)$. In liquid Ar the non-VUV emission spectrum was reported to be continuous [17, extending from 400 to $1000 \mathrm{~nm}$, in contrast to the atomic line spectrum in gaseous Ar. Accordingly, for liquid Ar we used the borosilicate glass filter transparent in both the NIR and visible range. Taking these facts into account, Fig. 3 shows the scintillation yield in liquid $\mathrm{Ar}$ at $87 \mathrm{~K}$, in the NIR and visible region, as a function of the electric field varying from 0 to $30 \mathrm{kV} / \mathrm{cm}$. For comparison, the NIR scintillation yield in gaseous $\mathrm{Ar}$ is also shown at the same temperature, i.e. in saturated vapor at $87 \mathrm{~K}$ and $1.0 \mathrm{~atm}$, as well as at $163 \mathrm{~K}$ and $0.60 \mathrm{~atm}$. The scintillation yield in Fig. 3 is given in the number of photons per $\mathrm{MeV}$ of deposited energy of the primary ionization, using a $\mathrm{W}$-value of 26.4 and $23.6 \mathrm{eV}$ for gaseous and liquid Ar respectively

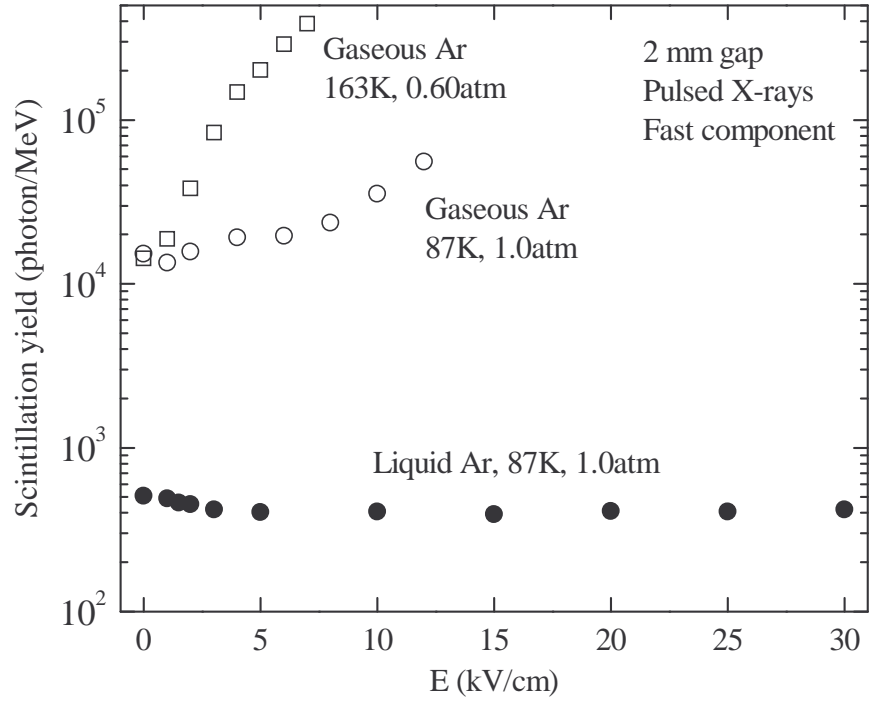

Fig. 3: Scintillation yield for the fast component as a function of the electric field in liquid Ar in the NIR and visible region, at $87 \mathrm{~K}$ and $1.0 \mathrm{~atm}$, and in gaseous $\mathrm{Ar}$ in the NIR, at $163 \mathrm{~K}$ and $0.60 \mathrm{~atm}$ and at $87 \mathrm{~K}$ and $1.0 \mathrm{~atm}$. The scintillation yield is given in the number of photons per $\mathrm{MeV}$ of deposited energy of the primary ionization. The data were obtained under X-ray irradiation in a $2 \mathrm{~mm}$ thick gap.

8. The primary scintillation yield in liquid Ar, defined at zero field, amounts to $Y_{L A r}=510 \pm 90$ photon/MeV. Notice that it is considerably reduced, by a factor of 30 , compared to that of gaseous Ar. The effect of suppression of NIR scintillations in the noble liquid as compared to that of the noble gas was observed earlier for Xe [15. The mechanism of such suppression is yet unclear.

From Fig. 3 one can see that the scintillation yield in gaseous Ar increased with the electric field starting from a certain threshold: in particular in $\mathrm{Ar}$ at $163 \mathrm{~K}$ and $0.60 \mathrm{~atm}$ - from $2 \mathrm{kV} / \mathrm{cm}$. This increase is explained by secondary scintillations (electroluminescence) induced by electrons drifting and exciting atoms under moderate electric fields, at which the gap is operated in an ionization mode, i.e. without electron multiplication. This kind of scintillations is called proportional scintillations due to the proportionality of their intensity to the electric field. The effect of proportionality of NIR scintillations in Ar was observed earlier at room temperature [16, though their absolute yield was not measured. In the present work, in gaseous Ar at $163 \mathrm{~K}$ the law of proportionality was valid up to $6 \mathrm{kV} / \mathrm{cm}$. At this field the scintillation yield increased by an order of magnitude compared to that of the primary scintillations, reaching a value of $200 \times 10^{3}$ photon/MeV (or 5 photon/e) for the effective gap thickness of $1 \mathrm{~mm}$. Consequently, we confirm here the high secondary NIR scintillation yield observed recently in our laboratory using THGEM multiplier 22: at avalanche gains of 60400 it was of the order of 4 photon/e.

In denser gaseous $\mathrm{Ar}$, at $87 \mathrm{~K}$ and $1 \mathrm{~atm}$, proportional scintillations were also observed, though at higher thresh- 


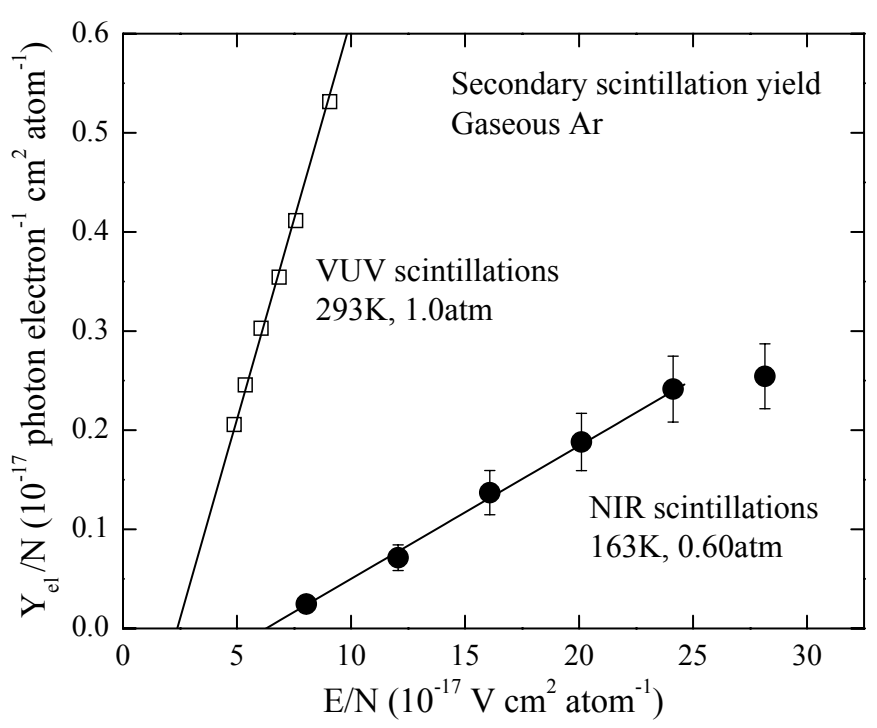

Fig. 4: Reduced NIR electroluminescence yield for the fast component as a function of the reduced electric field in gaseous Ar at $163 \mathrm{~K}$ and 0.60 atm measured in the present work; for comparison that of the VUV measured elsewhere 10 is shown.

old (above $8 \mathrm{kV} / \mathrm{cm}$ ). In liquid Ar in contrast, no proportional scintillations were observed at all, even at fields reaching $30 \mathrm{kV} / \mathrm{cm}$ (see Fig. 3).

The electroluminescence yield is defined as the number of photons normalized to the total ionization charge generated in the gap, the primary scintillation contribution being subtracted, and to the average electron drift path in the gap $(d)$, equal to $1 \mathrm{~mm}$ for the top gap:

$Y_{e l}=N_{p h} / N_{e} / d$.

To compare our electroluminescence results obtained in the NIR to those obtained in the VUV, the reduced electroluminescence scintillation yield is shown in Fig. 4 as a function of the reduced electric field. The linear part of the yield dependence corresponds to proportional scintillations; in the NIR at $163 \mathrm{~K}$ it is described by the following equation:

$Y_{e l} / N \quad\left[10^{-17}\right.$ photon electron ${ }^{-1} \mathrm{~cm}^{2} \quad$ atom $\left.^{-1}\right]=$ $0.013 E / N-0.084$.

Here $N$ is the atomic concentration and $E / N$ is given in $\mathrm{Td}\left[10^{-17} \mathrm{~V} \mathrm{~cm}^{2}\right.$ atom $\left.^{-1}\right]$. This equation is universally valid for any temperature and pressure. Thus it may be compared to the proportional scintillation yield in the VUV at room temperature presented elsewhere [10:

$Y_{e l} / N \quad\left[10^{-17}\right.$ photon electron ${ }^{-1} \mathrm{~cm}^{2}$ atom $\left.^{-1}\right]=$ $0.081 E / N-0.190$.

One can see that the electroluminescence amplification parameter in the NIR, defined as the number of photons produced per drifting electron and per kilovolt, equivalent to the line slope in Fig. 4 , is equal to 13; this is by a factor of 6 lower compared to that of the VUV. One can also see that proportional scintillations exist in the NIR up to reduced field values of $25 \mathrm{Td}$. At higher fields the gap started to operate in a proportional mode, i.e. with electron mul- tiplication, at which the atomic excitation processes are taken over by those of the avalanche ionization, resulting in the reduction of the electroluminescence efficiency.

Let us consider now possible applications of NIR scintillations. As mentioned in the Introduction, the first application is the THGEM-based two-phase Ar avalanche detector of ultimate sensitivity (i.e. operating in singleelectron counting mode at extremely low noise) with GAPD optical readout 22], for coherent neutrino-nucleus scattering and dark matter search experiments. The principles of operation of such a detector are shown in Fig. 5 . these are the further development of the concept suggested in [7] for coherent neutrino-nucleus scattering experiment. The ionization, produced by nuclear recoil from a weakly interacting particle, is extracted from the liquid into the gas phase, where it is amplified in two stages: first, in a proportional scintillation mode in a gap above the liquidgas interface, and second, in an avalanche scintillation mode in a THGEM multiplier. Proportional scintillations are recorded in the VUV and NIR using PMTs placed in the liquid: this will provide a single-photoelectron trigger due to the excellent amplitude resolution available in the proportional scintillation mode. Avalanche scintillations are recorded in the NIR using a matrix of bare G-APDs: this will provide a good spatial resolution. Such an optical readout is preferable as compared to charge readout in terms of overall gain and noise. In particular it has been demonstrated 22] that the THGEM/G-APD combined multiplier could operate in a single electron counting mode in a two-phase Ar avalanche detector, at an avalanche gain of 400. A practical detector would comprise a G-APDs matrix placed a few millimeters behind the THGEM multiplier, with a pitch of $1 \mathrm{~cm}$, viewing clusters of multiplier holes under an angle of $\pm 45^{\circ}$. For example, for a $100 \mathrm{~kg}$ liquid Ar TPC of a volume of $40 \times 40 \times 40 \mathrm{~cm}^{3}$ the total number of G-APDs would be reasonable - of 1600. Such a detector would be robust, stable, simple and relatively cheap.

The other application might be noble liquid non-VUV scintillation calorimetry. The scintillation yield in liquid Ar measured here in the NIR and visible region $(\sim 500$ photon/MeV) might be enough for high energy calorimetry: it is comparable with that of the fast solid scintillators being already used in calorimeters, namely higher than that of $\mathrm{PbWO}_{4}(\sim 100$ photon/MeV) and somewhat lower than that of pure CsI $(\sim 2000$ photon/MeV). The NIR scintillation yield in liquid $\mathrm{Xe}$ is expected to be of the same order as that of Ar, providing the applicability in liquid Xe NIR scintillation calorimetry. The readout of the calorimeters might be performed using NIR-sensitive photodetectors: silicon APDs and G-APDs for liquid Ar and InGaAs photodiodes for liquid Xe.

One more application is the project for directional solar neutrino detection using a GEM-based cryogenic Ne or He detector with CCD optical readout (so-called "e-bubble" project) 30. Here the property of intense NIR scintillations in noble gases is combined with that of the high 


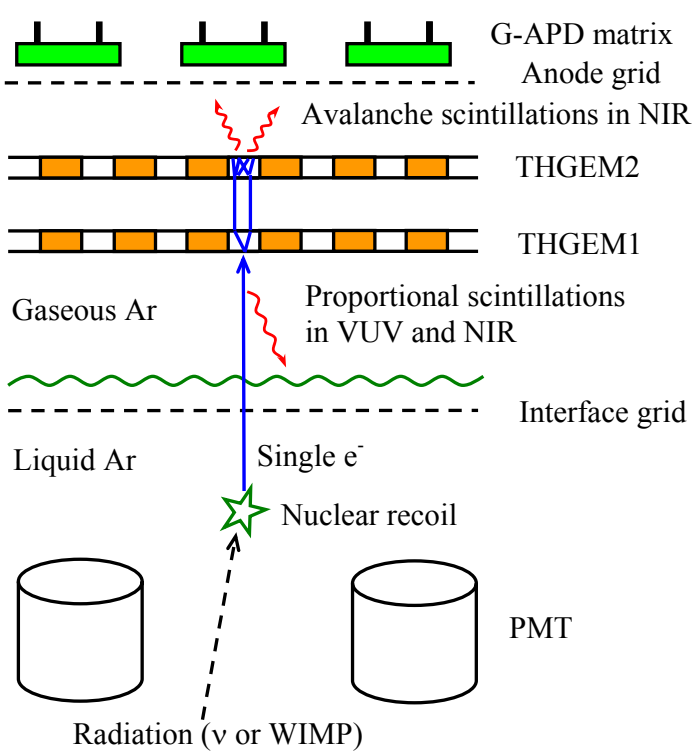

Fig. 5: Principles of operation of a THGEM-based two-phase Ar avalanche detector with G-APD optical readout for coherent neutrino-nucleus scattering and dark matter search experiments.

\section{CCD sensitivity in the NIR.}

Conclusion. - The scintillation yield in gaseous and liquid Ar has for the first time been measured in the NIR and visible region, both for primary and secondary (proportional) scintillations, using Geiger-mode avalanche photodiodes (G-APDs) and pulsed X-ray irradiation.

In gaseous Ar, the non-VUV scintillations took place essentially in the NIR: the primary scintillation yield of the fast component was measured to be $17000 \pm 3000$ photon/MeV or $0.44 \pm 0.09$ photon/e, in the range of $690-1000$ $\mathrm{nm}$. This is comparable with that of the VUV for gaseous Ar and Xe.

In liquid Ar, the primary scintillation yield of the fast component was considerably reduced, amounting to $510 \pm$ 90 photon/MeV in the range of 400-1000 nm. Nevertheless this is comparable with the yields of fast solid scintillators used in high-energy calorimetery.

Proportional scintillations (electroluminescence) in the NIR have been observed in gaseous Ar; their amplification parameter at $163 \mathrm{~K}$ was measured to be 13 photons per drifting electrons per $\mathrm{kV}$. Though having somewhat lower yield than that in the VUV, proportional scintillations may substantially increase the scintillation yield as compared to that of primary scintillations in the NIR: by an order of magnitude, to hundreds of thousands photons per $\mathrm{MeV}$.

No proportional scintillations have been observed in liquid Ar up to the electric fields of $30 \mathrm{kV} / \mathrm{cm}$.

There is potentially a wide variety of applications of noble gas NIR scintillations in high energy physics experiments. Among them are THGEM-based two-phase Ar avalanche detectors of ultimate sensitivity with G-APD optical readout for coherent neutrino-nucleus scattering and dark matter search experiments, liquid noble scintillation calorimetry, GEM-based cryogenic detectors with CCD optical readout for solar neutrino detection, and others.

$$
* * *
$$

We are grateful to Y. Tikhonov for the support and D. Akimov for discussions. This work was supported in part by Federal special program "Scientific and scientificpedagogical personnel of innovative Russia" in 2009-2013.

\section{REFERENCES}

[1] Akimov D.Yu. et AL., Astropart. Phys., 27 (2007) 46.

[2] Angle J. et Al., Phys. Rev. Lett., 100 (2008) 021303.

[3] Benetti P. et Al., Astropart. Phys., 28 (2007) 495.

[4] Adam J. et AL., Nucl. Phys. B, 834 (2010) 1.

[5] Diaz J. et Al., J. Phys. Conf. Ser., 179 (2009) 012005.

[6] Hagmann C. and Bernstein A., IEEE Trans. Nucl. Sci., 51 (2004) 2151.

[7] Akimov D. et AL., JINST, 4 (2009) P06010.

[8] Aprile E. and Doke T., Rev. Mod. Phys., 82 (2010) 2053.

[9] Aprile E., Bolotnikov A., Bolozdynya A. and Doke T., Noble gas detectors (WIILEY-VCH, Weinheim) 2006.

[10] Monteiro C.M.B. ET Al., Phys. Lett. B, 668 (2008) 167.

[11] Fernandes L.M.P. ET AL., JINST, 5 (2010) P09006.

[12] Suzuki M. and Kubota S., Nucl. Instr. Meth., 164 (1979) 197.

[13] Usenko A. et Al., Phys. Rev. Lett., 90 (2003) 153201.

[14] Lindblom P. and Solin O., Nucl. Instr. Meth. A, 268 (1988) 204

[15] Bressi G. et AL., Nucl. Instr. Meth. A, 461 (2001) 378.

[16] Bressi G. ET AL., Phys. Lett. A, 278 (2001) 280.

[17] Heindl T. et AL., Eur. Phys. Lett., 91 (2010) 62002.

[18] Fraga M.M. et AL., IEEE Trans. Nucl. Sci., 47 (2000) 933.

[19] Belogurov S. et AL., Nucl. Instr. Meth. A, 452 (2000) 167.

[20] Renker D. and Lorenz E., JINST, 4 (2009) P04004.

[21] CPTA company, http://www.cpta-apd.ru http://www.photonique.ch.

[22] BONDAR A. ET AL., JINST, 5 (2010) P08002.

[23] Bondar A. et Al., JINST, 3 (2008) P07001.

[24] Breskin A. Et. AL., Nucl. Instr. Meth. A, 598 (2009) 107.

[25] Lightfoot P.K. Et AL., JINST, 4 (2009) P04002.

[26] http://www.messer.ch

[27] Bondar A. ET AL., Nucl. Instr. Meth. A, 598 (2009) 121.

[28] Bondar A. ET AL., Nucl. Instr. Meth. A, 628 (2011) 364

[29] Buzulutskov A. ET AL., Near infrared scintillations in gaseous and liquid Ar, in preparation, to be submitted to JINST.

[30] Ju Y.L. ET AL., Cryogenics, 47 (2007) 81. 